\title{
Totally Tubeless Percutaneous Nephrolithotomy: A Comparison with Tubeless and Standard Methods
}

\author{
Ali Eslahi, ${ }^{1}$ Dariush Irani, ${ }^{2}$ Mohammad Mehdi Hosseini, ${ }^{2, *}$ and Ali Reza Safarpour ${ }^{3}$ \\ ${ }^{1}$ Shiraz Geriatric Research Center, Shiraz University of Medical Sciences, Shiraz, IR Iran \\ ${ }^{2}$ Shiraz Nephrology-Urology Research Center, Shiraz University of Medical Sciences, Shiraz, IR Iran \\ ${ }^{3}$ Colorectal Research Center, Shiraz University of Medical Sciences, Shiraz, IR Iran \\ "Corresponding author: Mohammad Mehdi Hosseini, M.D., Urology Office, Faghihi Hospital, Zand Blvd., Shiraz, IR Iran. Tel/Fax: +98-7132326645, E-mail: \\ mmhosseini@sums.ac.ir
}

Received 2017 April 17; Accepted 2017 May 24.

\begin{abstract}
Objectives: The study aimed to compare tubeless, totally tubeless, and standard percutaneous nephrolithotomy (PNL) in a tertiary center with selected patient population.

Methods: Between September 2013 and March 2015, percutaneous nephrolithotomy was performed on 216 patients in our center. Patients without massive bleeding, calyceal perforation, and residual fragments smaller than $4 \mathrm{~mm}$ were enrolled in this study. The patients were divided into 3 groups. In the first group $(n=68)$, we placed nephrostomy and kept ureteral catheter for 24 hours (the standard group). In second the group $(n=69)$, no nephrostomy was inserted and ureteral catheter was removed in the operation room (the totally tubeless group) and in the third group $(n=79)$, no nephrostomy catheter was placed but antegrade DJ stent was inserted intraoperatively (the tubeless group). Three groups were compared with respect to age, analgesic requirement, BMI, length of stay, pain, and stone size.

Results: No significant differences were found in the mean stone size, operation time, transfusion rates, and hemoglobin level change between the groups. However, hospitalization time, the amount of narcotic usage, and pain were significantly lower in the second group compared to the other groups $(\mathrm{P}<0.05)$.

Conclusions: In patients with no major intraoperative bleeding and calyceal perforation, the totally tubeless approach is safe with decreased pain, analgesia requirement, and hospital stay.
\end{abstract}

Keywords: Nephrolithiasis, Percutaneous, Tubeless, Outcome

\section{Introduction}

Since the introduction of Percutaneous nephrolithotomy (PCNL) in 1976, it has been rapidly considered as the standard of care for management of large renal stones ( 1 , 2 ). The standard method in PCNL is nephrostomy tube placement after surgery for draining the kidney. Postoperative complications by premature nephrostomy tube removal has been reported in original PCNL studies $(3,4)$. Most urologists do PCNL in a standard manner in their practice but stent and drainage tube-related pain is a common complaint among patients; therefore, modern techniques have been developed to perform PCNL without nephrostomy drainage called tubeless PCNL $(2,5,6)$. A newer approach named totally tubeless PCNL is a procedure without the placement of a nephrostomy or ureteral catheter, which has showed reasonable results in selected cases (7-9). In the present retrospective study, we compared the possibility and limits of tubeless, totally tubeless PCNL, and standard method in our tertiary referral center.

\section{Methods}

Between September 2013 and March 2015, we retrospectively analyzed the data of 216 patients who underwent PCNL at our center and fulfilled the inclusion criteria of the study. Patients without massive bleeding, calyceal system perforation, and residual fragments smaller than 4 mm were enrolled in this study. Those with single kidney, more than two accesses, supracostal access, large staghorn stones, and kidneys with congenital anomalies or patients with urosepsis were excluded.

Preoperative radiological evaluation was ultrasonography and noncontrast computed tomography scan for delineating the kidney anatomy and renal stone configuration. All patients had either negative urine culture or received preoperative antibiotic based on organism sensitivity. All PCNL procedures were done under general anesthesia in prone position. Briefly, after ureteric catheterization, access to the target calyx was made under guide of fluoroscopy. After tract dilation and nephroscopic evaluation, pneumatic lithotripter was used for stone fragmentation. Endoscopic and fluoroscopic evaluations were used for as- 
sessment of stone free status and any residual fragment.

Patients were classified into three categories: group 1 (standard method) ( $\mathrm{n}=68)$ : nephrostomy (Fr 18) was inserted and ureteral catheter was kept in place for 24 hours. Group 2 (totally tubeless) $(n=69)$ : no nephrostomy was inserted and the ureteral catheter was removed after completion of the surgery. Group 3 (tubeless) $(n=79)$ no nephrostomy was inserted but a double $\mathrm{j}$ ureteral stent was used and kept in place for 10 days after the procedure. Hematocrit was checked six hours after the surgery on a daily basis. For postoperative analgesia, the intramuscular pethidine HCL on demand was given to patients. Patients were evaluated by kidney ureter bladder (KUB) films and ultrasonography postoperatively. Patients with residual fragments were referred for ancillary procedures (e.g., shock wave lithotripsy (SWL) or ureteroscopy). The decision to remove the nephrostomy in the standard group was based on postoperative radiography or ultrasonography as well as urine color.

Age, BMI, analgesic need, pain according to linear visual score, changes in hemoglobin level after the operation, stone size and location, length of hospitalization, and complications according to Clavien-Dindo grading system were compared between the three groups.

\subsection{Statistical Analysis}

Quantitative factors were demonstrated as mean \pm SD, and qualitative variables were shown as frequency and percentage. One way analysis of variance and post hoc test and Kruskal-Wallis test were performed as needed.

All analyses were performed using SPSS version 16 (Inc. Chicago, IL). P-values above 0.05 were considered significant.

\subsection{Informed Consent}

Informed consent regarding the type of procedure, advantages, disadvantages, and possible complications was obtained from all individual participants included in the study.

\section{Results}

Demographic characteristics of the patients participating in the three study groups are presented in Tables 1 and 2. Table 1 presents quantitative data and Table 2 presents qualitative variables.

All the three groups were compared in terms of independent variables. The ANOVA was performed to compare the quantitative variables between the three groups. The results of these analyses showed a significant difference between the three groups in the variables of pain, length of stay in hospital, and taking painkillers after surgery $(\mathrm{P}<$ $0.001, \mathrm{P}<0.001$, and $\mathrm{P}=0.04$, respectively)

These three groups did not show any significant difference in other remaining variables $(\mathrm{P}=0.03)$.

LSD Post Hoc test showed that the standard and tubeless groups showed a significant difference in the variable of using "painkillers after surgery" $(\mathrm{P}=0.03)$.

In addition, the standard and no tubeless groups showed a significant difference with the totally tubeless group in the variables of pain and length of stay $(\mathrm{P}<$ 0.001).

The frequency of the location of kidney stones for the three groups is presented in Table 3.

Complications induced in the three groups based on the scoring method are presented in Table 4 . There was no significant difference between the three groups in terms of complications $(\mathrm{P}=0.79)$.

\section{Discussion}

In the present research, PCNL was done in three groups with different techniques concerning tubes and stent insertion. We compared discomfort levels and complication rates in the context of how to use tubes and stents in PCNL procedure. The placement of nephrostomy tube after PCNL is considered as a standard method to fulfill multiple purposes including hemostasis, adequate drainage of the collecting system, and allowing further access for the second look; but, it can also cause discomfort, pain, and prolonged hospitalization (10). The use of the ureteral stents has also some disadvantages such as lower urinary tract symptoms (LUTS) and cystoscopy requirement for its removal that imposes additional costs.

Recently, several methods such as avoiding nephrostomy or ureteral stents insertion (tubeless and completely tubeless PCNL) that have been described to decrease the postoperative pain, hospitalization, and morbidity of the patients (11).

The safety and efficacy of tubeless PCNL has been shown in several reports (12-14). As reported by Desai et al., the size of nephrostomy can affect postoperative morbidity. They compared small (9F) vs. conventional (20F) tube after PCNL and showed that patients with 9F nephrostomy tube had less pain and hospital stay in comparison with $20 \mathrm{~F}$ tube patients (13). Karami et al. reported their 5year experience with tubeless PCNL in 201 patients and indicated that it can be done safely in kidney stones larger than $2 \mathrm{~cm}$ as well as in staghorn stones (15). In a review article that was done by Zilberman et al. it was shown that tubeless PCNL has some advantages such as less pain, less debilitation, less costs, and a quicker recovery in all patient 
Table 1. Patients Characteristics, Quantitative Variables ${ }^{\mathrm{a}}$

\begin{tabular}{|c|c|c|c|c|}
\hline \multirow[t]{2}{*}{ Variable } & \multicolumn{3}{|c|}{ Group } & \multirow[t]{2}{*}{ PValue $^{\text {b }}$} \\
\hline & Standard & Totally Tubeless & Tubeless & \\
\hline Age & $46.47(1.45)$ & $44.2(1.5)$ & $49.45(1.34)$ & 0.083 \\
\hline BMI & $26.06(4.43)$ & $25.78(4.68)$ & $26.32(4.24)$ & 0.759 \\
\hline Stone Size, cm & $2.56(1.18)$ & $2.09(0.89)$ & $2.38(0.89)$ & 0.021 \\
\hline Cr Change & $0.32(2.4)$ & $0.16(1.37)$ & $0.26(2.09)$ & 0.897 \\
\hline Hb Change & $1.69(1.14)$ & $1.85(0.97)$ & $1.63(1.59)$ & 0.979 \\
\hline pain & $7.19(1.14)$ & $4.19(0.92)$ & $6.01(0.84)$ & $<0.001$ \\
\hline Length of stay & $56.12(11.99)$ & $38.09(10.68)$ & $48.91(9.37)$ & $<0.001$ \\
\hline Analgesic, mg & $73.76(59.13)$ & 52.99 (97.99) & $50.65(19.97)$ & 0.043 \\
\hline
\end{tabular}

${ }^{\mathrm{a}}$ Values expressed as No. (\%)

${ }^{\mathrm{b}} \mathrm{P}$ value calculated by ANOVA test.

Table 2. Patient Characteristics, qualitative Variables ${ }^{\mathrm{a}}$

\begin{tabular}{|c|c|c|c|c|c|}
\hline \multirow[t]{2}{*}{ Variable } & & \multicolumn{3}{|c|}{ Group } & \multirow[t]{2}{*}{ PValue $^{\mathrm{b}}$} \\
\hline & & Standard & Totally Tubeless & Tubeless & \\
\hline \multirow{2}{*}{ Sex } & Male & $49(37.4)$ & $40(30.5)$ & $42(32.1)$ & \multirow{2}{*}{0.056} \\
\hline & Female & $19(22.4)$ & $29(34.1)$ & $37(43.5)$ & \\
\hline \multirow{2}{*}{ laterality } & RT & $29(28.4)$ & $32(31.4)$ & $41(40.2)$ & \multirow{2}{*}{0.526} \\
\hline & LT & $39(34.2)$ & $37(32.5)$ & $38(33.3)$ & \\
\hline \multirow{2}{*}{ Recurrence } & Yes & $19(28.4)$ & $22(32.8)$ & $26(38.8)$ & \multirow{2}{*}{0.796} \\
\hline & No & $49(32.9)$ & $47(31.5)$ & $53(35.6)$ & \\
\hline \multirow{2}{*}{ Fever } & Yes & $10(62.5)$ & $1(6.2)$ & $5(31.2)$ & \multirow{2}{*}{0.011} \\
\hline & No & $58(29)$ & $68(34)$ & $74(37)$ & \\
\hline
\end{tabular}

${ }^{\mathrm{a}}$ Values expressed as No. (\%).

${ }^{\mathrm{b}} \mathrm{P}$ value calculated by kruskal wallis test.

Table 3. Frequency Location of Stones in three Groups ${ }^{\mathrm{a}}$

\begin{tabular}{lccc}
\hline Variable & \multicolumn{3}{c}{ Group } \\
\cline { 2 - 4 } & Standard & Totally Tubeless & Tubeless \\
\hline Lp* & $18(23.7)$ & $39(51.3)$ & $19(25)$ \\
\hline Mp** & $13(32.5)$ & $8(20)$ & $19(47.5)$ \\
\hline Up*** & $6(28.6)$ & $7(33.3)$ & $8(38.1)$ \\
\hline Staghhorn & $11(34.4)$ & $7(21.9)$ & $14(43.8)$ \\
\hline Pelvic & $17(41.5)$ & $6(14.6)$ & $18(43.9)$ \\
\hline Lp and Pelvic & $1(50)$ & $1(50)$ & 0 \\
\hline Up and Lp & $1(100)$ & 0 & 0 \\
\hline
\end{tabular}

${ }^{\mathrm{a}}$ Values expressed as No. (\%).
Table 4. Frequency of Complications in Three Groups ${ }^{\mathrm{a}}$

\begin{tabular}{lccc}
\hline Complications $^{\mathbf{b}}$ & \multicolumn{3}{c}{ Groups } \\
\cline { 2 - 4 } & Standard & Totally Tubeless & Tubeless \\
\hline No Complication & $58(85.3)$ & $61(88.4)$ & $70(88.6)$ \\
Grade 1 & $7(10.3)$ & $6(8.7)$ & $6(7.6)$ \\
\hline Grade 2 & $2(2.9)$ & $1(1.4)$ & $3(3.8)$ \\
Grade 3 & $1(1.5)$ & $1(1.4)$ & - \\
\hline
\end{tabular}

${ }^{\mathrm{a}}$ Values expressed as No. (\%).

${ }^{\mathrm{b}}$ Grading according to Clavian grading System (10).

groups including children and obese patients (16). Istanbulluoglu et al. used an externalized ureteral catheter instead of double $\mathrm{j}$ stent in tubeless patients to reduce the morbidity caused by stents and to omit the requirement 
of cystoscopy for its removal (17).

In our opinion, we can use externalized ureteral catheters in tubeless PCNL patients when there are no suspicious migrated stones into ureter during the surgery. Therefore, performing tubeless PCNL seems an intraoperative decision. In the current research, we used double $\mathrm{j}$ stents in our tubeless patients to ensure ureteral patency after the operation.

Wickham et al. were the first who reported totally tubeless PCNL in one hundred cases without either internal or external drainage tubes with outcome (18).

Totally tubeless PCNL without the use of nephrostomy or ureteral catheters has been popularized since 2004 (19). Crook et al. compared totally tubeless PCNL with standard method in a randomized trial; patients in the group of totally tubeless were selected based on intraoperative findings such as intact calyceal system, no residual stones, and no evidence of bleeding. They reported a $96 \%$ stone free rate, reduced hospital stay to 2.3 days, and a lower analgesia need for the patients with totally tubeless method (20).

In another study by Crook et al. totally tubeless PCNL was considered a safe process in selected patients. Although hemorrhage and ureteral obstruction were the main concerns in totally tubeless procedure, they showed that lack of tube can stop bleeding from the tract (9).

Similar results were reported by Karami and Gholamrezaie through in a comparative analysis of totally tubeless versus standard PCNL (21).

In highly selected patients, totally tubeless PCNL can be remarked as a method with reduced morbidity.

PCNL is a challenging operation with a complication rate of $1.1 \%-7 \%$ in experienced hands. The main problem is hemorrhage that can happen during any stage of the procedure. Placing a nephrostomy may help avoid this complication (22). However, in our series, the mean hemoglobin drop, blood transfusion need, and the overall complication rate were similar in all the three groups.

We used the modified Clavien system to report surgical complication rates (23). Controversial rates of complications have been reported in different series. While some trials showed a lower rate of complications in totally tubeless PCNL (24), others reported a similar rate of complications $(17,21)$.

In our experience, the decision on how to perform PCNL is dependent on intraoperative findings. Major intraoperative bleeding and pelvicalyceal system perforation preclude performing totally tubeless PCNL. We believe that in our study, patients were selected appropriately for totally tubeless procedure because there are no more complications in this group of patients. Totally tubeless PCNL should not be done in patients having residual stones because it precludes second look nephroscopy and it may be ended up with significant urinary leakage.

\subsection{Conclusions}

Totally tubeless PCNL has less postoperative discomfort, hospital stay, and morbidity with no added complications if patients are selected appropriately.

\section{Acknowledgments}

Authors would like to thank Dr. Alireza Aminsharif for his kind cooperation in preparing the draft of the manuscript.

\section{Footnotes}

Funding/Support: This study was funded by Shiraz Nephro-urology research center, Shiraz University of Medical Sciences, Shiraz, Iran.

Conflict of Interest: All authors declare that they have no conflict of interest in this study.

Ethical Approval: The study was in accordance with ethical standards of Shiraz University of Medical Sciences and national research committee.

\section{References}

1. Fernstrom I, Johansson B. Percutaneous pyelolithotomy. A new ex traction technique. Scand J Urol Nephrol. 1976;10(3):257-9. [PubMed: 1006190].

2. Delnay KM, Wake RW. Safety and efficacy of tubeless percutaneous nephrostolithotomy. World J Urol. 1998;16(6):375-7. [PubMed: 9870282].

3. Winfield HN, Weyman P, Clayman RV. Percutaneous nephrostolithotomy: complications of premature nephrostomy tube removal.JUrol. 1986;136(1):77-9. [PubMed: 3712621].

4. Abbott JE, Deem SG, Mosley N, Tan G, Kumar N, Davalos JG. Are we fearful of tubeless percutaneous nephrolithotomy? Assessing the need for tube drainage following percutaneous nephrolithotomy. Urol Ann. 2016;8(1):70-5. doi: 10.4103/0974-7796.162214. [PubMed: 26834406].

5. Agrawal MS, Agrawal M, Gupta A, Bansal S, Yadav A, Goyal J. A randomized comparison of tubeless and standard percutaneous nephrolithotomy. J Endourol. 2008;22(3):439-42. doi: 10.1089/end.2007.0118. [PubMed:18257738].

6. Beiko D, Lee L. Outpatient tubeless percutaneous nephrolithotomy: the initial case series. Can Urol Assoc J. 2010;4(4):E86-90. [PubMed: 20694090].

7. Gupta V, Sadasukhi TC, Sharma KK, Yadav RG, Mathur R. Tubeless and stentless percutaneous nephrolithotomy. BJU Int. 2005;95(6):905-6. doi: 10.1111/j.1464-410X.2005.05432.x. [PubMed: 15794808].

8. Chang $\mathrm{CH}$, Wang $\mathrm{CJ}$, Huang SW. Totally tubeless percutaneous nephrolithotomy: a prospective randomized controlled study. Urol Res. 2011;39(6):459-65. doi: 10.1007/s00240-011-0363-0. [PubMed: 21331773].

9. Crook TJ, Lockyer CR, Keoghane SR, Walmsley BH. Totally tubeless percutaneous nephrolithotomy. J Endourol. 2008;22(2):267-71. doi: 10.1089/end.2006.0034. [PubMed: 18095863]. 
10. Bellman GC, Davidoff R, Candela J, Gerspach J, Kurtz S, Stout L. Tubeless percutaneous renal surgery. J Urol. 1997;157(5):1578-82. [PubMed: 9112480].

11. Shah HN, Kausik VB, Hegde SS, Shah JN, Bansal MB. Tubeless percutaneous nephrolithotomy: a prospective feasibility study and review of previous reports. BJU Int. 2005;96(6):879-83. doi:10.1111/j.1464410X.2005.05730.x. [PubMed: 16153222].

12. Sofikerim M, Demirci D, Huri E, Ersekerci E, Karacagil M. Tubeless percutaneous nephrolithotomy: safe even in supracostal access. Endourol. 2007;21(9):967-72. doi: 10.1089/end.2006.0216. [PubMed: 17941770].

13. Desai MR, Kukreja RA, Desai MM, Mhaskar SS, Wani KA, Patel SH et al. A prospective randomized comparison of type of nephrostomy drainage following percutaneous nephrostolithotomy: large bore versus small bore versus tubeless. J Urol. 2004;172(2):565-7. doi: 10.1097/01.ju.0000130752.97414.c8. [PubMed: 15247731].

14. Limb J, Bellman GC. Tubeless percutaneous renal surgery: review of first 112 patients. Urology. 2002;59(4):527-31. [PubMed: 11927306] discussion 531.

15. Karami H, Jabbari M, Arbab AH. Tubeless percutaneous nephrolithotomy: 5 years of experience in 201 patients. $J$ Endourol. 2007;21(12):1411-3. doi: 10.1089/end.2007.0406. [PubMed: 18052821].

16. Zilberman DE, Lipkin ME, de la Rosette JJ, Ferrandino MN, Mamoulakis C, Laguna MP, et al. Tubeless percutaneous nephrolithotomythe new standard of care? J Urol. 2010;184(4):1261-6. doi 10.1016/j.juro.2010.06.020. [PubMed: 20723920].

17. Istanbulluoglu MO, Cicek T, Ozturk B, Gonen M, Ozkardes H. Percutaneous nephrolithotomy: nephrostomy or tubeless or totally tube- less? Urology. 2010;75(5):1043-6. doi: 10.1016/j.urology.2009.06.104. [PubMed: 19854490].

18. Wickham JE, Miller RA, Kellett MJ, Payne SR. Percutaneous nephrolithotomy: one stage or two? Br J Urol. 1984;56(6):582-5. [PubMed: 6534470].

19. Aghamir SM, Hosseini SR, Gooran S. Totally tubeless percutaneous nephrolithotomy. $J$ Endourol. 2004;18(7):647-8. doi: 10.1089/end.2004.18.647. [PubMed: 15597653].

20. Crook TJ, Lockyer CR, Keoghane SR, Walmsley BH. A randomized controlled trial of nephrostomy placement versus tubeless percutaneous nephrolithotomy. J Urol. 2008;180(2):612-4. doi: 10.1016/j.juro.2008.04.020. [PubMed: 18554657].

21. Karami H, Gholamrezaie HR. Totally tubeless percutaneous nephrolithotomy in selected patients. J Endourol. 2004;18(5):475-6. doi: 10.1089/0892779041271580. [PubMed: 15253824].

22. Rastinehad AR, Andonian S, Smith AD, Siegel DN. Management of hemorrhagic complications associated with percutaneous nephrolithotomy. J Endourol. 2009;23(10):1763-7. doi: 10.1089/end.2009.1548. [PubMed:19747040].

23. Dindo D, Demartines N, Clavien PA. Classification of surgical complications: a new proposal with evaluation in a cohort of 6336 patients and results of a survey. Ann Surg. 2004;240(2):205-13. [PubMed: 15273542].

24. Moosanejad N, Firouzian A, Hashemi SA, Bahari M, Fazli M. Comparison of totally tubeless percutaneous nephrolithotomy and standard percutaneous nephrolithotomy for kidney stones: a randomized, clinical trial. Braz J Med Biol Res. 2016;49(4):e4878. doi: 10.1590/1414431X20154878. [PubMed: 27007650]. 